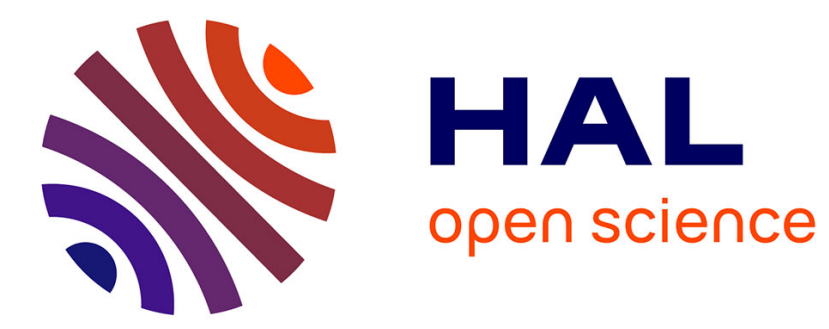

\title{
Fin de la guerre froide, fin de l'état de guerre?
}

Dario Battistella

\section{To cite this version:}

Dario Battistella. Fin de la guerre froide, fin de l'état de guerre?. Politique étrangère, 1993, 58 (3), pp.747-761. 10.3406/polit.1993.6285 . halshs-00111150

\section{HAL Id: halshs-00111150 https://shs.hal.science/halshs-00111150}

Submitted on 26 Apr 2019

HAL is a multi-disciplinary open access archive for the deposit and dissemination of scientific research documents, whether they are published or not. The documents may come from teaching and research institutions in France or abroad, or from public or private research centers.
L'archive ouverte pluridisciplinaire HAL, est destinée au dépôt et à la diffusion de documents scientifiques de niveau recherche, publiés ou non, émanant des établissements d'enseignement et de recherche français ou étrangers, des laboratoires publics ou privés. 


\section{Fin de la guerre froide, fin de l'état de guerre ?}

\section{Battistella}

\section{Citer ce document / Cite this document :}

Battistella. Fin de la guerre froide, fin de l'état de guerre ?. In: Politique étrangère, $\mathrm{n}^{\circ} 3-1993-58^{\mathrm{e}}$ année. pp. 747-761; doi : https://doi.org/10.3406/polit.1993.6285

https://www.persee.fr/doc/polit_0032-342x_1993_num_58_3_6285

Fichier pdf généré le 13/04/2018 


\title{
Résumé
}

Depuis la chute du mur de Berlin le monde assiste une prolifération de conflits tous azimuts Pourtant en ce qui concerne les relations entre Etats du Centre la fin de la guerre froide signifie abord le remplacement progressif un système international hétérogène par un système homogène la remise en question de la priorité de la politique de puissance par la politique influence ainsi que impossibilité croissante établir une séparation entre politique interne et politique étrangère avènement un tel système-monde transnational inédit implique certes pas la fin des relations de domination dépendance entre Etats-nations Mais il en met pas moins en cause les postulats traditionnels du paradigme réaliste de état de guerre

\begin{abstract}
End of the Cold War End of the State of War by Dario Battistella Since the falling down of the Berlin Wall there is proliferation of interna tional conflicts throughout the world But for the central states the end of the Cold War above all means the progressive substitution of heterogeneous international system by homogeneous one the greater importance of influence politics in comparison to traditional power politics as well as the growing impossibility of strictly separating domestic and foreign policies Of course the rise of such an unknown transnational world-system does not put an end to international domination and dependency But it probably means that the classical state-of-war-paradigm is no longer appropriate to contempo rary international relations
\end{abstract}




\section{libre propos}

\section{\begin{tabular}{l|l} 
Dario BATtISTELLA * & $\begin{array}{l}\text { Fin de la guerre froide, } \\
\text { fin de l'état de guerre? }\end{array}$
\end{tabular}}

$\mathrm{L}$

a fin de la guerre froide a remis en cause un certain nombre de concepts auxquels recouraient les spécialistes de politique internationale pour analyser celle-ci depuis la fin de la Seconde Guerre mondiale système bipolaire, équilibre de la terreur, Tiers-Monde, etc. Désireux de remplacer ces notions par des concepts et des outils d'analyse susceptibles de saisir la nouvelle donne internationale, les chercheurs en relations internationales ont dû se contenter rapidement de définitions la plupart du temps négatives, comme le prouvent les expressions telles que "le grand désordre mondial " [1], les «troubles " ou les «illusions" du nouvel ordre [2], ou "l'ordre mondial relâché " [3].

S'il en est ainsi, c'est parce que la fin de la guerre froide n'est nullement interprétée comme signifiant le retour de l'harmonie, le triomphe de la raison hégélienne ou l'instauration de la paix perpétuelle kantienne: tout au contraire, et malgré la rhétorique du nouvel ordre mondial cher au président Bush, les experts sont d'accord pour estimer que "l'ordre international va plutôt vers des manifestations d'éclatement que vers l'idéal d'une communauté policée " [4]. Plus particulièrement, le risque de voir un nouveau désordre, un nouveau chaos des nations se substituer à l'ordre de Yalta est considéré comme d'autant plus grand que de nouvelles menaces et de nouveaux dangers sont à l'ordre du jour [5]. On a même l'impression d'assister à une course de vitesse pour trouver coûte que coûte un ersatz au traditionnel facteur de guerre potentiel qu'était l'opposition Est-Ouest, un ennemi de substitution susceptible de prendre le relais de l'ancien empire du mal qu'était l'Union soviétique [6], tant l'après-guerre froide, caractérisée par "le transitoire, l'instable, le désarticulé et l'ambivalent "[7], voire par "le primat de la turbulence et $d u$ désordre $[8]$, défie toute analyse rationnelle.

\section{Réalités nouvelles, concepts anciens}

Autrement dit, les notions remises en cause sont des notions conjoncturelles, liées aux bouleversements et à l'accélération de l'histoire contemporaine, et non des concepts transhistoriques susceptibles de servir d'outils d'analyse intemporels. En effet, substituer la notion de multipolarité à celle de bipolarité pour définir la nature du système international, faire succéder les conflits identitaires aux affrontements idéologiques comme principale source des ten-

* Chargé de mission au Service d'information et de diffusion du Premier ministre.

L'auteur s'exprime à titre personnel. 
sions contemporaines, ou voir dans le jeu économico-culturel plutôt que dans le jeu militaro-stratégique le principal échiquier de l'après-guerre froide, ne met pas en cause la croyance en ce que la politique étrangère reste fondamentalement une politique de puissance et que les relations entre Etats continuent de constituer un état de guerre.

Peu importe à ce sujet le scénario d'avenir privilégié par les uns ou les autres. Qu'il s'agisse de la crainte d'un retour à un système multipolaire intrinsèquement perçu comme plus instable que l'affrontement bipolaire de la guerre froide, parce qu'associé au système ayant abouti aux deux grandes guerres mondiales [9] ; qu'il s'agisse de la perspective d'un regain des tensions économiques entre grandes puissances occidentales du fait de la disparition de l'ennemi commun soviétique [10]; ou qu'il s'agisse du scénario de l'avènement d'un empire unipolaire hégémonique que seraient les Etats-Unis restés seule superpuissance [11] ; nous avons chaque fois affaire à la même conception de la politique internationale considérée comme étant "en tant que telle politique de puissance "[12] - power politics, Machtpolitik - et comme se déroulant par définition dans un environnement anarchique.

Si l'on peut certes comprendre la volonté de s'accrocher à un cadre d'analyse connu, et donc rassurant [13], à un moment où les ruptures successives multiplient les inconnues, la permanence d'une vision considérant les relations internationales comme des rapports a priori conflictuels $n$ 'est cependant pas au-dessus de tout soupçon : une telle perception reste en effet tributaire d'une vision philosophico-politique qui date, au delà de l'époque de la guerre froide, de l'ère classique de la diplomatie occidentale, celle du système interétatique consacré par les traités de Westphalie de 1648, sans parler de l'époque de la cité-Etat grecque lorsque fut esquissée pour la première fois, bien avant l'avènement de l'Etat territorial moderne, l'idée de l'inéluctabilité de la guerre entre unités politiques voisines, donc rivales.

Or, si l'on compare les tendances internationales à l'œuvre en cette fin de $\mathrm{XXe}$ siècle avec les réalités connues par les théoriciens de l'état de guerre en question, alors il ne fait guère de doute que, des quatre postulats réalistes que sont l'unité politique souveraine acteur exclusif sur la scène politique internationale, la primauté de la politique de puissance dans les relations entre unités politiques, la séparation stricte entre politique interne et environnement politique international, la présence d'un système international hétérogène opposant deux messianismes irréductibles, aucun n'est plus pertinent aujourd'hui.

Ne nous attardons pas sur le premier point : il est admis aujourd'hui que, si l'Etat reste l'acteur principal de la scène internationale, il n'en est cependant plus l'acteur exclusif, du fait de la prolifération d'un nombre impressionnant d'acteurs infra-, supra-, et non étatiques [14]. Plus intéressants sont déjà les trois autres constats qui montrent que, même à l'intérieur de la sphère internationale qui continue de relever du contrôle de l'acteur étatique juridiquement souverain, les changements intervenus sont tels que continuer de raisonner en termes de politique de puissance et d'état de guerre n'a plus guère de sens pour ce qui est des relations qu'entretiennent entre elles les principales puissances.

La première critique que l'on peut adresser au paradigme classique peut en effet être établie à partir de la simple observation de la vie politique 
internationale contemporaine : contrairement à la grande majorité des rapports interétatiques du passé, les relations entre Etats du Centre - que l'on peut définir, par opposition aux Etats périphériques [15], comme des unités politiques disposant des capacités leur permettant d'accéder, au moins partiellement, aux marchés de production des biens matériels et symboliques constitutifs de la puissance internationale - ont cessé d'être caractérisées par la primauté de la puissance politico-militaire et ne se déroulent plus à l'ombre de la guerre. L'erreur en la matière consiste précisément à ne pas voir que ce que l'on a appelé le "retour de l'histoire" dans les relations entre les républiques de l'ex-URSS et l'ex-Yougoslavie est probablement un dernier soubresaut qui ne saurait faire oublier la progressive pacification des relations internationales contemporaines.

\section{Etat de guerre et politique de puissance}

L'idée que la politique menée entre elles par les unités politiques serait essentiellement une politique de puissance, débouchant logiquement et régulièrement sur des affrontements guerriers, date de l'historiographie grecque : c'est en effet Thucydide qui, le premier, dans sa narration de la guerre du Péloponnèse, a posé le postulat de l'état de guerre, en attribuant la cause ultime de la guerre opposant Athènes à Sparte au fait que "les Athéniens, en s'accroissant, donnèrent de l'apprébension aux Lacédémoniens, les contraignant ainsi à la guerre» $[16]$.

En affirmant que les républiques "vivent dans un état de guerre perpétuelle, dans une continuelle veillée d'armes, leurs frontières fortifiées, leurs canons braqués sur tous les pays qui les entourent " [17], Thomas Hobbes systématisera cette idée que Machiavel avait déjà reprise, en notant qu'il est impossible pour une République "de demeurer tranquille et de jouir paisiblement de sa liberté. En effet, si elle n'attaque pas ses voisins, elle sera attaquée par eux, et cette attaque lui inspirera l'envie de conquérir et l'y forcera malgré elle "[18]. Il finira, logiquement, par hypostasier l'état de guerre : " $A$ tous moments les rois et les personnes qui détiennent l'autorité souveraine sont, à cause de leur indépendance, dans une continuelle suspicion, et dans la situation et position des gladiateurs, leurs armes pointées, les yeux de chacun fixés sur l'autre (...), toutes choses qui constituent une attitude de guerre $*[19]$.

Après avoir été consacrée tour à tour par Rousseau, Hegel et Clausewitz, cette approche, dite réaliste, des relations internationales sera remise au goût de la guerre froide par les réalistes américains, Hans Morgenthau notamment, et, en France, par Raymond Aron, tous les deux convaincus de la permanence de la guerre. "La guerre est de tous les temps historiques et de toutes les civilisations", écrit ainsi Raymond Aron [20], rejoint en cela par Hans Morgenthau, pour qui "la lutte pour la puissance est universelle dans le temps et dans l'espace" [21]. Enfin, la gauche se convertira elle aussi aux vertus de la politique de puissance, à l'image d'un Régis Debray qui, devenu conseiller du prince, se propose comme objectif de détourner les siens de l'orthodoxie socialiste en matière de relations internationales : faite de croyances illusoires en la paix par l'arbitrage, le désarmement et la collectivité, celle-ci aurait en effet le tort d'oublier la brutalité inhérente aux rapports internationaux, le fait primordial que "la puissance est la substance même des relations internationales" [22]. 
Ce que l'on peut remarquer chez l'ensemble de ces auteurs, c'est qu'au delà de leurs a priori métaphysiques sur l'animus dominandi inhérent à la nature humaine, ils partagent tous la même expérience politique fondamentale : celle d'avoir vécu à des époques d'intense activité militaire. Autrement dit, on peut supposer que c'est leur Seinsgebundenheit (solidarité situationnelle) qui explique leur acharnement à voir dans les rapports entre unités politiques des relations substantiellement martiales, à ressentir cette condition conflictuelle comme un phénomène normal, voire naturel, et non plus politique, et à la rationaliser dans le concept d'état de guerre consistant en la guerre de tous contre tous. C'est vrai pour Thucydide, les Grecs ayant successivement connu les guerres médiques, la guerre du Péloponnèse, la guerre contre la Macédoine ; et c'est tout aussi vrai pour Thomas Hobbes, témoin tout à la fois de la guerre civile anglaise, de la guerre entre l'Angleterre et l'Ecosse et de la guerre de Trente Ans.

Mais c'est également vrai pour Hans Morgenthau, Raymond Aron et Régis Debray. La guerre froide dont ils ont été les témoins a en effet, tout en excluant tout affrontement direct entre les deux principaux protagonistes, multiplié, par alliés et satellites interposés, des conflits provoquant la mort de 40 millions de personnes. Par ailleurs et surtout, l'équilibre de la terreur caractéristique de ces quarante années constitue l'idéal-type de l'«attitude de guerre" chère à Thomas Hobbes. Si l'on sait que, pour ce dernier, "la guerre ne consiste pas seulement dans la bataille et les combats effectifs, mais dans un espace de temps où la volonté de s'affronter est suffisamment avérée"; si l'on sait que, pour lui, "la nature de la guerre ne consiste pas dans un combat effectif́, mais dans une disposition avérée, allant dans ce sens, aussi longtemps qu'il n'y a pas d'assurance $d u$ contraire "[23], alors on peut comprendre que, pour les adeptes modernes de la realpolitik, la paix chaude de l'après-Seconde Guerre mondiale ait pu être considérée comme une simple "suspension, plus ou moins durable, des modalités violentes de la rivalité entre unités politiques" [24], regroupées en l'occurrence dans le camp occidental et dans le bloc soviétique.

Reste à savoir si cette accentuation unilatérale de l'état de guerre latent n'est pas excessive. Le prétendu réalisme de Thomas Hobbes n'était déjà qu'une des interprétations possibles des réalités politiques de son époque. C'est ce que permettent de penser a contrario les écrits de Grotius et Locke qui, quoi que datant de ce même XVIIe siècle, partent de l'hypothèse d'un état de nature, défini par contraste à l'état de guerre hobbésien comme n'étant "nullement un état de licence" [25], tant les relations de coopération et de détente qui se nouent entre unités politiques souveraines le disputent aux conflits qui peuvent les opposer par ailleurs.

De nos jours, et jusqu'à la chute du mur de Berlin, la prévalence du facteur politico-militaire sur les autres logiques ne saurait certes faire aucun doute. Mais de là à dire, comme n'hésite pas à le faire Régis Debray, que "L'arbre des conventions de Genève (...) cache la verte forêt des génocides, agressions, occupations, représailles, terrorisme et chantage " [26], c'est oublier que depuis la fin de la Seconde Guerre mondiale, et tout au long de la guerre froide, aucune des principales puissances ne s'est trouvée imbriquée dans un conflit mettant en cause son intégrité territoriale ni même son indépendance nationale : mieux même, la période sans guerre que l'Europe vit depuis 1945 est la 
plus longue qu'elle ait jamais connue depuis l'avènement du système étatique postmédiéval [27].

Depuis la fin de la guerre froide, cette tendance des Etats centraux à ne plus connaître concrètement, physiquement, si l'on peut dire, l'état de guerre - à moins de considérer comme constitutif d'un état de guerre des expéditions aussi limitées dans le temps et/ou dans l'espace que Suez, les Malouines, le Vietnam ou le Golfe - a encore été accentuée, et ce, parce que le primat du facteur politico-militaire s'érode au fur et à mesure, premièrement, que s'estompe la menace d'une guerre et, deuxièmement, que la puissance strictement militaire est de plus en plus frappée de non-fongibilité.

Traditionnellement considérée comme l'étalon de mesure par excellence du rang d'une unité politique dans le concert des nations, la force brute, strictement militaire, perd progressivement de son utilité politique. L'émergence de l'arme nucléaire $a$, à ce sujet, joué un grand rôle. Pour s'en convaincre, il suffit de rappeler cette vérité trop souvent oubliée lorsque l'on fait des Etats-Unis et de l'ex-URSS les deux superpuissances par excellence de l'histoire de l'humanité : en effet, le fait pour les deux ex-supergrands de la guerre froide de disposer d'une capacité potentielle de destruction infiniment supérieure à toutes les puissances du passé, n'a pas empêché la diminution de leur puissance réelle au sens de capacité d'imposer leur volonté, comme l'ont tour à tour démontré - pour ne citer que quelques exemples -, d'un côté l'évolution des relations entre l'URSS et la Chine, ou entre les Etats-Unis et la France, et, de l'autre, le sort auquel ont abouti l'intervention de la première en Afghanistan, et des seconds au Vietnam.

\section{Power politics ou influence politics?}

Mais l'existence d'une arme nucléaire non opérationnelle parce qu'utilisable à des fins exclusivement dissuasives, donc défensives - c'est ce que l'on peut appeler le dilemme nucléaire : on ne peut se battre ni avec ni contre des armes nucléaires - n'est pas seule en cause. Car, au delà du coût exorbitant - aucune cause ne mérite l'autodestruction - qu'entraînerait le recours en premier à l'arme nucléaire, c'est le ratio coût/bénéfice de l'ensemble de la panoplie militaire qui devient de nos jours trop aléatoire, tant les bénéfices politiques que la possession de la force brute est susceptible d'entraîner se révèlent de plus en plus illusoires [28].

Pour preuve, à un niveau très ponctuel, la guerre du Golfe qui, si elle a permis d'atteindre les objectifs limités fixés aux alliés par les Nations Unies, en l'occurrence le rétablissement de la souveraineté du Koweit, n'a en rien réglé les problèmes politiques qui continuent de se poser au Proche et Moyen-Orient. Pour preuve aussi, sur un plan plus général, le fait que la domination politico-militaire exercée par les Etats-Unis sur l'Allemagne et le Japon, les deux perdants de la Seconde Guerre mondiale, n'a pas empêché ces derniers de sortir vainqueurs de la guerre froide [29].

Dans ce processus de dévalorisation de l'échiquier politico-stratégique, l'importance grandissante prise par les relations économiques internationales - il faudrait plutôt dire transnationales - a joué le rôle principal. Ainsi, l'analyse marxiste a-t-elle pu croire un moment que la disparition, depuis la fin de la Seconde Guerre mondiale, des traditionnels affrontements " interimpérialistes " était due à l'avènement de l'ennemi commun soviétique [30] : 
face à ce dernier, les alliés occidentaux faisaient prévaloir non pas leurs intérêts nationaux, mais leur intérêt commun. Autrement dit, la disparition de cette épée de Damoclès, en faisant ressortir les divergences d'intérêts, était censée sonner le glas de la coopération intra-occidentale et relancer les traditionnelles contradictions intercapitalistes. Or, force est de constater que ce raisonnement pêche par excès de simplisme.

Non pas qu'une telle argumentation surestimerait l'importance des oppositions d'intérêts économiques, mais elle méconnaît sûrement la nature de l'échiquier sur lequel se joue désormais l'essentiel de la vie internationale : en effet, si l'interdépendance économique n'est en elle-même nullement synonyme de baisse de tensions, au contraire, ces tensions n'en sont pas moins de moins en moins violentes [31]. Et ce, parce que, sur le terrain de la compétition géoéconomique, les civilian powers [32] que sont de plus en plus les Etats du Centre préfèrent mettre davantage l'accent sur le soft power que sur le hard power [33]. Certes, les deux sortes de relations de puissance s'inscrivent dans une dialectique de la domination et de la dépendance, dans la mesure où, conformément à la définition webérienne de la puissance, les capacités, sinon d'imposer son influence à autrui, du moins de se soustraire à celle d'autrui, relèvent toutes les deux d'une logique du commandement et de l'obéissance. Mais le passage insensible de la traditionnelle power politics, qui consiste en un recours à la force coercitive pour imposer sa volonté à un autre pays, à la pratique de l'influence politics [34], qui consiste en un recours aux moyens non violents que sont la persuasion et le marchandage, n'est pas sans conséquence sur la nature profonde des relations entre unités politiques et du système international global qui en découle.

Si en effet dans le monde transnational, c'est-à-dire économiquement interdépendant, de l'après-guerre froide, la puissance d'un Etat s'exprime moins dans sa volonté à imposer sa politique à ses partenaires-rivaux, que dans sa capacité à persuader ces derniers à se joindre à la politique qu'il préconise, ou du moins à pratiquer une politique compatible avec ses intérêts, alors c'est la substance même de la politique internationale qui subit un processus de transformation : car, s'il est vrai que les tensions continuent d'exister, elles sont bien davantage résolues dans les salles de négociation et devant des cours d'arbitrage que durant des réunions d'états-majors ou sur les champs de bataille. Autrement dit, le conflit international, que l'on peut définir comme un affrontement entre deux unités politiques cherchant à se neutraliser, à s'affaiblir ou à s'éliminer l'une l'autre dans la perspective de l'obtention d'un avantage en termes de richesse, de puissance ou de gloire, perd de son acuité, étant donné que le recours à la décision ultime, celle qui consiste à faire usage du jus belli, en lequel réside précisément, à en croire Raymond Aron, la spécificité des relations entre unités politiques souveraines, tombe progressivement en désuétude, remplacé par des marathons de négociations où se succèdent propositions et contrepropositions, chantages et concessions, ruptures et reprises. Et ce, d'autant plus que, contrairement à ce qui passait dans le passé, il n'existe plus guère de corrélation étroite entre puissance économique et puissance politique.

Dans la perspective mercantiliste de l'économie qui a longtemps prévalu parmi les spécialistes des relations internationales s'intéressant à l'élément économique de la puissance, tôt ou tard une puissance économique était condamnée à s'aventurer sur le terrain politique : c'est notamment la thèse 
qui sous-tend le best-seller de Paul Kennedy sur le déclin progressif des Etats-Unis [35] face à la puissance économique montante de la CE et du Japon. Il est vrai que, par le passé, la puissance économique tendait logiquement vers la puissance politique, en ce que le principe du libre-échange, fondement de la puissance économique dans un marché mondial, n'a pu en dernier recours être imposé aux récalcitrants que grâce à la force militaire, comme a dû s'en rendre compte, à ses dépens, le Japon confronté au siècle dernier aux vaisseaux du Commodore Perry; de même que, de façon plus générale, une solide base économique a été la condition sine qua non d'un cycle d'hégémonie politique, comme l'a attesté le rôle joué par les interactions entre domination politico-militaire et productivité économique dans l'avènement et la disparition des empires.

\section{Transnationalisme vs. mercantilisme}

Mais, de là à conclure à l'existence d'une équation simpliste puissance économique $=$ puissance politico-militaire, il y a un pas que l'on aurait tort de franchir trop hâtivement, et ce, à cause de la difficile transférabilité de la force économique sur le terrain politique.

Tout d'abord, la nature du pouvoir économique, financier, technologique, diffère fondamentalement de celle du pouvoir diplomatico-militaire : " $\mathrm{Ce}$ pouvoir naît des interactions entre entreprises ayant lieu sur un marché fondamentalement transnational, voire global, ce qui milite contre l'idée d'une manipulation consciente des capacités économiques dans un but politique " [36]. Il s'ensuit très logiquement que la puissance économique n'est plus, de nos jours, un outil politique facile à manier par les autorités politiques, et ce, parce que "cette force (économique) représente la combinaison d'un ensemble de ressources relevant majoritairement de décideurs privés nombreux et souvent non (nationaux), en d'autres termes difficilement mobilisables en vue d'une action d'initiative gouvernementale " [37].

Cette nature différente de la puissance économique conduit à une remise en cause de la traditionnelle loi d'airain du nécessaire expansionnisme des puissances impérialistes. Cette loi, formulée déjà par Thucydide considérant qu'Athènes était condamnée à agrandir constamment son empire sur les autres cités-Etats grecques si elle voulait maintenir sa prépondérance, et érigée en principe de conduite diplomatique par Thomas Hobbes — "on ne peut pas rendre sûrs, sinon en en acquérant davantage, le pouvoir et les moyens dont dépend le bien-être que l'on possède présentement " [38] -, est typique du raisonnement en termes de somme nulle caractéristique du paradigme réaliste. Or, de nos jours, la nature transnationale et intangible du facteur économique, ainsi que l'abandon de toute logique mercantiliste - je perds ce que tu gagnes et vice-versa - , aboutissent à supprimer l'une des principales sources traditionnelles de conflits entre puissances, à savoir la conquête territoriale, dans la mesure où la conquête des parts de marché a rendu inutile la recherche de l'agrandissement territorial direct ou indirect, c'est-à-dire la possession d'empires coloniaux, et ce, malgré l'importance que l'existence de zones économiques exclusives pourrait avoir dans un avenir où l'exploitation des fonds marins prendrait le relais de l'exploitation des ressources naturelles en voie d'épuisement. 
Ensuite et surtout, la recherche de la richesse économique peut constituer un but en soi, et non un moyen en vue de contrôler autrui : "Si l'on en croit l'exemple de l'Allemagne et du Japon, voire de la CEE dans son ensemble, le premier but de ceux qui en disposent peut être parfois d'en jouir, pas de contrôler les autres " [39]. Apparemment naïve, une telle hypothèse n'est pas sans avoir des implications théoriques de premier plan. Quiconque s'est intéressé à l'histoire des idées et/ou à la formation comparée des Etats modernes sait en effet qu'à l'origine du passage des monarchies absolutistes aux régimes parlementaires, la revendication des satisfactions matérielles de la part des exclus du pouvoir a joué un grand rôle : c'est en effet du mouvement des Levellers et Diggers britanniques qu'est parti le processus qui, un demi-siècle plus tard, et sur la base de la remise en cause d'un pouvoir absolutiste ayant pour objectif le seul intérêt d'Etat défini en termes de sécurité et de grandeur, a abouti à la fois à la libéralisation de la vie politique et à la satisfaction des intérêts matériels des sujets devenus citoyens. Il n'est pas impossible que cette dimension, trop souvent oubliée, soit de nouveau à lordre du jour. Ce serait là une nouvelle réfutation portée au paradigme réaliste.

Celui-ci, n'accordant son intérêt qu'à la structure du système international défini en termes de rapports de forces, part en effet de l'hypothèse de l'acteur étatique personnifié en ses dirigeants, hypothèse considérée comme *une fiction nécessaire et un préalable indispensable à tout raisonnement et à toute discussion argumentés en relations internationales "[40]. Qu'il s'agisse de Thucydide décelant derrière les rituels démocratiques de la vie politique athénienne l'habileté manœuvrière de Périclès [41]; qu'il s'agisse de Thomas Hobbes faisant l'éloge du représentant-souverain qui, institué par les individus en vue du bien commun de ces derniers, doit pouvoir passer outre à leurs intérêts privés [42]; ou qu'il s'agisse de Raymond Aron, selon qui, étant donné "les passions des foules (et) les intransigeances idéologiques, il faut regretter que les Etats ne ressemblent pas davantage à des personnes et non déplorer la personnification de l'Etat " [43] ; tous les réalistes partagent volontiers ce pessimisme anthropologique qui les conduit à assumer spontanément que "les affaires internationales, après tout, consistent en des relations entre gouvernements, et non entre peuples", et que par conséquent, dans les matières relevant de la politique étrangère, "l'opinion publique ne saurait avoir la même influence qu'en politique interne " [44].

Or, un tel postulat, dont les origines remontent à la formation des Etats territoriaux postmédiévaux et à l'apparition du concept moderne de souveraineté au sens de capacité de commandement emportant obéissance, et qui par là même conforte l'exclusion des citoyens de tout contrôle et, a fortiori, de toute participation à la conduite de la politique extérieure, ne tient plus la route aujourd'hui. Et ce, parce que, du fait de la progressive interpénétration des scènes politiques interne et externe, les responsables politiques ne peuvent plus prendre leurs décisions en fonction des seuls impératifs de politique externe, indépendamment de toute prise en compte des demandes internes. Tout au contraire, l'interdépendance entre l'économie nationale et le marché mondial, ainsi que le fait que l'on soit passé de l'Etat-gendarme à l'Etatprovidence, les oblige à tout faire pour limiter les effets néfastes des tensions internationales sur la vie économique interne [45]. Et quoi de plus néfaste précisément qu'un conflit guerrier dont les effets seraient ressentis à tous les niveaux de la société ? 
Déjà, comme l'ont montré les enquêtes d'opinion menées avant l'entrée en action des alliés contre l'Irak, conflit que l'on pouvait pourtant, a priori, considérer comme ayant une portée limitée [46], il est devenu impossible aujourd'hui de déclencher une guerre frisch und fröblich, fraîche et joyeuse. $A$ fortiori, la guerre entre puissances majeures est-elle de moins en moins envisagée comme un recours possible pour régler les conflits d'intérêts, non seulement parce qu'elle a été reconnue comme étant contreproductive, et que par conséquent elle est perçue comme un "luxe" que seuls les pauvres peuvent se permettre, mais aussi et surtout parce qu'elle est devenue inimaginable, voire impensable dans le subconscient d'une population au sein de laquelle une longue période d'absence de guerre a suscité la naissance d'attentes pacifiques [47].

Contrairement au postulat réaliste qui ne tient compte que de la structure du système international et de la répartition de la puissance entre unités politiques, et non de l'orientation politico-idéologique des acteurs étatiques qui en sont les protagonistes, la dynamique interne d'un Etat influe par conséquent sur la nature d'un système international considéré à tort comme étant, en soi, anarchique. Sur un plan théorique, le triple processus de modernisation qu'a connu l'Etat - au niveau politique, on est passé d'un Etat de sujets à un Etat de citoyens ; au niveau économique, on est passé d'un Etat autarcique à un Etat interdépendant ; au niveau symbolique, on est passé d'un Etat chargé de garantir la seule sécurité physique à un Etat chargé d'assurer la sécurité économique et même sociale - implique alors la rupture avec le postulat de la nécessaire autonomie de la politique internationale par rapport à l'ordre politique interne des unités politiques et la prise en compte de l'évolution des régimes politiques internes [48].

Justement, si l'on part de l'hypothèse que l'anarchie internationale, loin d'être inscrite dans la structure des relations interétatiques, n'est jamais que ce que les Etats en font [49], alors la fin de la guerre froide, synonyme d'universalisation de la double légitimité démocratie-économie de marché, entraîne une troisième rupture avec les postulats sous-jacents au paradigme réaliste. Dans la mesure où l'impossibilité pour les décideurs de faire abstraction de l'environnement interne est d'autant plus grande que la légitimité des gouvernements est liée à leur capacité à satisfaire les intérêts particuliers de leurs électeurs, la progressive diffusion du régime de la démocratie libérale de marché a en effet d'importantes conséquences sur la substance du comportement politique des Etats sur la scène internationale : il est de moins en moins facile de persuader la classe moyenne urbanisée du bien-fondé d'une politique extérieure aventurière, parce qu'elle est de plus en plus consciente des conséquences que cette dernière pourrait avoir sur ses intérêts en termes de niveau de vie.

\section{Vers un système international homogène}

Par-delà des corrélations excessivement optimistes établies par Kant entre démocratie interne et paix extérieure, il ne fait ainsi guère de doute que l'expansion de la démocratie est, a priori, favorable à une diminution de la dimension guerrière de la vie internationale - depuis 1945 d'ailleurs, les démocraties occidentales ont mis sur pied une véritable zone de paix séparée entre elles, aucune des démocraties existantes, parmi lesquelles une superpuis- 
sance et plusieurs grandes puissances, n'ayant mené une guerre contre une autre démocratie.

Et ce, pour plusieurs raisons concernant en premier lieu la nature du régime démocratique. En effet, allant de pair avec l'économie de marché, la démocratie tend à favoriser les relations économiques internationales dont la croissance est bien évidemment directement fonction de la stabilité et de la prévisibilité des relations politiques. Par ailleurs, les démocraties se caractérisent par un degré élevé de mobilité de leurs ressortissants, mobilité favorable à la relativisation des valeurs nationalistes et à l'homogénéisation des mœurs, voire à l'émergence d'une culture mondiale partagée au moins par les élites des pays concernés.

Mais l'accession à la modernité politique joue également un rôle indirect, concernant cette fois-ci la nature du système international, dans la diffusion de relations interétatiques plus pacifiques. Une lecture attentive des prémisses de la théorie réaliste telle qu'elle s'est exprimée successivement chez Thucydide, Thomas Hobbes et Raymond Aron, permet en effet de constater que pour tous les trois, la notion d'état de guerre est strictement liée à ce que Raymond Aron a appelé un système international hétérogène, composé d'Etats "organisés selon des principes différents et se réclamant de valeurs contradictoires ", se distinguant d'un système international homogène, composé d'Etats qui "appartiennent au même type, et obéissent à la même conception de la politique "[50].

En effet, nous avons dans les trois cas affaire à l'hétérogénéité d'un système international caractérisé par l'opposition irréductible entre deux messianismes inconciliables : démocratie/oligarchie opposant chez Thucydide Athènes à Sparte ; absolutisme/parlementarisme opposant chez Thomas Hobbes les partisans des Stuarts à ceux de Cromwell ; libéralisme/socialisme opposant chez Raymond Aron les alliés occidentaux au camp soviétique. Autrement dit, la vision de l'état de guerre n'est pas concevable sans cette dialectique du combat à mort entre deux systèmes politico-idéologiques rivaux luttant pour imposer leur conception de l'obligation politique.

Or, ici encore, la fin de l'ordre de Yalta entraîne une révision théorique. Sans aller jusqu'à croire avec Francis Fukuyama que la "fin de l'bistoire " [51] est devant la porte, on peut estimer qu'aujourd'hui "la démocratie de marché constitue la matrice du monde, la problématique légitime du système international " [52], avec pour conséquence la disparition des traditionnelles lignes de rupture coupant le monde en deux camps opposés.

D'un côté en effet, et seulement deux ans après la chute du mur de Berlin, le socialisme scientifique, qui, depuis un siècle et demi, prétendait représenter une force antisystème au libéralisme dominant, apparaît au pire comme une aberration, au mieux comme une parenthèse historique. On ne voit aucun affrontement global Nord-Sud succéder au conflit Est-Ouest, les intérêts en jeu étant trop mal définis et les camps en présence, le "Sud » bien plus encore que le «Nord", n'existant pas en tant qu'entités organisées en vue d'une action délibérée.

De l'autre, pour ce qui est des modèles politico-idéologiques potentiellement concurrents qui émergent, il leur manque soit l'un soit l'autre des deux éléments qui composent tout messianisme téléologique prétendant détenir la 
clef de l'énigme du monde. C'est le cas tout d'abord de l'islam : seule force antisystème relativement organisée en vue de produire un sens depuis que le tiers-mondisme a lui aussi disparu corps et biens, il ne constitue guère un rival capable de lancer un défi durable à l'hégémonie uniformisatrice du modèle occidental, et ce, non seulement parce que le message qu'il est susceptible de livrer n'exerce aucun pouvoir de séduction sur les élites ni sur les masses du centre, mais aussi parce que cette capacité à produire un sens universaliste ne s'accompagne d'aucune capacité à générer une puissance politico-économique. C'est le cas également du Japon, mais pour la raison inverse : principal rival potentiel intra-système, l'empire du Soleil Levant, s'il possède indéniablement la capacité technologico-financière indispensable à la production d'une puissance économique et militaire de tout premier plan, a jusqu'à nos jours été incapable de penser le monde et, qui plus est, s'est, depuis l'ère Meiji, date de son entrée dans le temps mondial, contenté du rôle d' importateur de sens »[53] et n'a jamais été tenté de diffuser son expérience nationale de développement comme modèle culturel universel.

Par conséquent, l'avènement d'un système international homogène, ou conservateur [54], devrait logiquement contribuer à une diminution sensible des rapports de puissance militaro-stratégique dans le monde de l'après-guerre froide. Car, en principe, le sentiment d'appartenance à une même communauté, l'existence d'institutions supranationales et de flux de communications transnationaux et d'interactions économiques, ainsi que le partage de valeurs à portée universelle, font que la traditionnelle politique de puissance cède, de facto, le pas dans les relations interétatiques contemporaines. Davantage, c'est l'équation classique "Etat = machine de guerre " [55], sinon la catégorie fondatrice de la politique internationale, à savoir la distinction schmittienne entre ami et ennemi [56], qui sont progressivement remises en cause.

Paradoxalement, c'est la guerre du Golfe, pourtant déploiement de force brute s'il en est, qui a apporté à ce sujet un début de preuve de ce que nous avançons. Bien qu'interprétée ici ou là comme exemple-type des conflits Nord-Sud à venir, elle ne s'inscrit en effet nullement dans le scénario classique de l'état de guerre des réalistes, mais souligne tout au contraire le renouveau de ce que l'on pourrait appeler, par opposition à la conception hobbésienne, la conception grotienne de la société internationale. Et ce, pour les raisons suivantes.

Dans un premier temps, l'invasion du Koweit par l'Irak peut se justifier, du point de vue de Saddam Hussein, par les dogmes les plus classiques de la diplomatie traditionnelle — "Là où il n'est pas de pouvoir commun, il n'est pas de loi; là où il n'est pas de loi, il (...) n'existe pas de propriété, pas d'empire sur quoi que soit, pas de distinction du tien et du mien : cela seul dont il peut se saisir appartient à chaque homme, et seulement pour aussi longtemps qu'il peut le garder"[57]. Autrement dit, nous sommes toujours en plein paradigme réaliste.

Mais, dans un second temps, la réaction des Etats non directement concernés par ce conflit est symptomatique des changements en cours : si la vision traditionnelle de la politique internationale comme politique de puissance avait continué de prévaloir dans l'air du temps, personne ne serait venu au secours du Koweit. Or, apparemment, la mobilisation internationale sans précédent indique qu'un changement de mentalité a peut-être eu lieu et que le 
droit, voire le devoir, reconnu par les jusnaturalistes à tous les peuples d'intervenir contre "une nation malfaisante " n'a pas définitivement abdiqué face à la loi du plus fort. Même si des considérations traditionnelles d'équilibre régional des puissances ont évidemment joué un rôle dans l'esprit des dirigeants occidentaux, on peut légitimement espérer qu'à la base de leur décision d'entrer en guerre contre l'Irak de Saddam Hussein il y ait également eu la conviction qu'il existe de nos jours une société composée d'Etats qui "reconnaissent certains intérêts, voire certaines valeurs, communs; qui s'estiment tenus de respecter l'indépendance des uns par rapport aux autres; qui pensent devoir honorer les engagements auxquels ils ont souscrits; et qui croient en la nécessité d'assujettir à certaines limites l'exercice de la force " [58]. On aurait alors affaire à l'abandon du dogme du droit au libre recours à la force au sein d'un système international anarchique, et au retour à la notion de guerre juste [59] qui, employée à titre défensif et en vue de recouvrer une propriété légitime, est utilisée comme sanction ultime à l'encontre d'un membre récalcitrant de ce qui est perçu comme une société, sinon des nations, du moins entre nations.

\section{Conclusion}

$\mathrm{Si}$ cette tendance à voir en les relations internationales moins un état de guerre qu'un état de détente [60] devait se confirmer, alors l'évolution en cours pourrait induire, au delà de l'atténuation du primat de la politique de puissance politico-militaire, la remise en cause de l'autonomie même du politique telle qu'elle avait été consacrée depuis Machiavel. C'est dans ce sens que l'on peut interpréter le renouveau, non seulement des valeurs utilitaristes, s'exprimant dans les rapports économiques, mais aussi, et surtout, des valeurs juridico-éthiques : renaissance du droit international - éthique des droits de l'homme, réactivation de l'ONU, reconnaissance d'un droit d'ingérence face au principe jadis tout-puissant de la non-ingérence dans les affaires intérieures d'un Etat ; retour en vogue d'une éthique de la responsabilité -, primauté du souci de survie de ce que d'aucuns n'hésitent pas à appeler la " société civile mondiale ", confrontée à l'émergence des biens communs [61].

Dans tous les cas, si l'on ne peut certes pas déduire de la fin de la guerre froide l'existence de relations harmonieuses entre Etats, il ne fait pas moins guère de doute que le contenu de ces relations a connu un changement significatif. Il est certes encore trop tôt pour déterminer s'il s'agit d'un changement de nature ou seulement de degré ou, pour parler en termes théoriques, si nous sommes en présence de développements susceptibles d'être à l'origine d'un changement de système ou dans le système [62], d'un changement d'ordre paramétrique ou de simples fluctuations d'ordre non paramétrique [63]. Mais le fait que n'existe plus de nos jours, dans les relations entre principales puissances, cette menace permanente d'un recours à la force et, a fortiori, d'une escalade à l'extrême, nous incite à ne voir dans la conduite diplomatico-stratégique chère à Raymond Aron qu'un élément parmi d'autres des relations internationales contemporaines, ni plus, ni moins. 


\section{NOTES BIBLIOGRAPHIQUES}

[1] Serge July, "Le grand désordre mondial ", dans La nouvelle planète, World-Media/Libération, Paris, décembre 1990, p. 3.

[2] Stanley Hoffmann, "A New World and Its Troubles», Foreign Affairs, vol. 69, $\mathrm{n}^{\circ} 4$, automne 1990, p. 115-122; et "Les illusions de l'ordre mondial ", Esprit, août-septembre 1992, p. 88-105.

[3] Zaki Laïdi, L'ordre mondial relâché. Sens et puissance après la guerre froide, Presses de la FNSP, Paris, 1993, p. 30.

[4] Bertrand Badie et Marie-Claude Smouts, Le retournement du monde. Sociologie de la scène internationale, Presses de la FNSP, Paris, 1992, p. 12.

[5] Voir par exemple Pierre Lellouche, Le nouveau monde. De l'ordre de Yalta au désordre des nations, Grasset, Paris, 1992.

[6] Voir par exemple l'article particulièrement suggestif de C. Krauthammer, "One Source of the New World Disorder Is Tehran ", International Herald Tribune, 4 janvier 1993. Tout aussi révélateur est le scénario d'histoire-fiction imaginé par The Economist des 26 décembre $1992-$ 8 janvier 1993 qui prévoit pour le siècle prochain la naissance, suite à un coup d'Etat fondamentaliste en Arabie, d'un empire islamique envahissant les Balkans et n'hésitant pas à s'allier à la Chine pour, ensemble, dépecer l'ex-Union soviétique.

[7] Zaki Laïdi, L'ordre mondial relâché, op. cit., p. 30.

[8] Pierrre Hassner, «L'épreuve de faiblesse ", Libération, 23 août 1991.

[9] Voir notamment l'article de J. Mearsheimer, "Back to the Future. Instability in Europe After the Cold War ", International Security, vol. 15, n 1, été 1990, p. 5-56, qui s'appuie lui-même sur les conclusions de K. Waltz, Theory of International Politics, Addison-Wesley, Boston, 1979.

[10] Voir par exemple I. Wallerstein, "Die posthegemoniale Ara ", dans Die neue Weltunordnung, World-Media/Die Tageszeitung, 24 décembre 1990, p. 8-9.

[11] C. Krauthammer, "The Unipolar Moment ", Foreign Affairs, vol. 70, n 1, America and the World 1990-1991, p. 23-33.

[12] C'est la définition donnée par Raymond Aron, Paix et guerre entre les nations, CalmannLévy, Paris, 1984, p. 132.

[13] Voir par exemple le dossier de la revue américaine The National Interest, $\mathrm{n}^{\circ} 30$, hiver 1992 1993, avec des articles de F. Zakaria, R. Tucker, O. Harries et R. Fox.

[14] Voir à ce sujet J. Rosenau, Turbulence in World Politics. A Theory of Change and Continuity, Princeton University Press, Princeton (N.J.), 1990, qui, après avoir établi une distinction entre "le monde interétatique " et "le monde multicentrique ", conclut à l'émergence d'un "monde mixte" où s'interpénètrent les actions des acteurs étatiques et des acteurs non étatiques.

[15] Pour une application du couple conceptuel centre-périphérie au monde de l'après-guerre froide, voir J. Goldgeier et M. McFaul, "A Tale of Two Worlds. Core and Periphery in the Post-Cold War Era ", International Organization, vol. 46, $n^{\circ} 2$, printemps 1992, p. 467-491.

[16] Thucydide, Histoire de la guerre du Péloponnèse, Robert Laffont, Paris, 1991, Livre premier, chapitre XXIII-6, p. 184.

[17] Thomas Hobbes, Léviathan, Sirey, Paris, 1971, chapitre XXI, p. 227.

[18] Nicolas Machiavel, "Discours sur la première décade de Tite-Live ", dans CEuvres complètes, Gallimard-La Pléiade, Paris, 1952, Livre second, chapitre XIX, p. 567.

[19] Thomas Hobbes, Léviathan, op. cit., chapitre XIII, p. 126.

[20] Raymond Aron, Paix et guerre entre les nations, op. cit., p. 157.

[21] Hans Morgenthau, Politics Among Nations. The Struggle For Power and Peace, Knopf, New York, 1973, p. 34

[22] Régis Debray, La puissance et les rêves, Gallimard, Paris, 1985, p. 131.

[23] Thomas Hobbes, Léviathan, op. cit., chapitre XIII, p. 124.

[24] Raymond Aron, Paix et guerre entre les nations, op. cit., p. 158.

[25] J. Locke, Traité du gouvernement civil, Flammarion, Paris, 1984, p. 174. 
[26] Régis Debray, La puissance et les rêves, op. cit., p. 42.

[27] C'est ce que rappelle C. Kaysen, "Is War Obsolete?", International Security, vol. 14, ${ }^{\circ} 4$, printemps 1990, p. 42-64.

[28] Voir à ce sujet les réflexions de Mark W. Zacher, "The Decaying Pillars of the Westphalian Temple. Implications for International Order and Governance ", dans J.N. Rosenau et E.O. Czempiel (sous la direction de), Governance Without Government. Order and Change in World Politics, Cambridge University Press, Cambridge (Mass.), 1992, p. 58-101.

[29] Voir à ce propos l'étude de K. Valaskakis, "Qui a gagné la guerre froide ? Du capitalisme des cowboys au capitalisme d'équipe ", dans C.P. David (sous la direction de), La fin de la guerre froide, Centre québéquois des relations internationales/Fondation pour les études de défense nationale, Québec/Paris,1990, p. 39-52.

[30] Voir par exemple l'analyse de D. Senghaas, "Formes et types de conflits dans la société internationale contemporaine", dans T. Hentsch et alii (sous la direction de), Le systeme mondial. Rapports internationaux et relations internationales, Nouvelle Optique, Montréal, 1983, p. 258-292.

[31] Sur l'impact de l'interdépendance économique sur la nature conflictuelle ou non des relations interétatiques, voir l'étude de M. Gasiorowski, "Economic Interdependance and International Conflict. Some Cross-National Evidence ", International Studies Quarterly, vol. 30, $\mathrm{n}^{\circ}$, mars 1986, p. 23-38.

[32] Nous empruntons cette expression à $H$. Maull, "Germany and Japan. The New Civilian Powers ", Foreign Affairs, vol. 70, n⿳ 5, hiver 1990-1991, p. 91-106.

[33] Distinction établie par Joseph Nye, Bound To Lead. The Changing Nature of American Power, Basic Books, New York, 1989.

[34] Distinction établie par A. Wolfers, Discord and Collaboration, Johns Hopkins University Press, Baltimore, 1962.

[35] Paul Kennedy, Naissance et déclin des grandes puissances, Payot, Paris, 1989.

[36] H. Maull, "Germany and Japan. The New Civilian Powers", article cité.

[37] Karl Kaiser, "Germany's Unification ", dans Foreign Affairs, vol. 70, n 1, janvier 1991, p. 179-205.

[38] Thomas Hobbes, Léviathan, op. cit., chapitre XI, p. 96.

[39] Bertrand Badie et Marie-Claude Smouts, Le retournement du monde, op. cit., p. 153.

[40] E.H. Carr, The Twenty Years' Crisis, MacMillan, Londres, 1946, p. 151.

[41] Selon Thucydide, Histoire de la guerre du Péloponnèse, op. cit., Livre second, chapitre LXV$8 / 9$, p. 280 , Périclès " avait de l'autorité grâce à la considération dont il jouissait, et à ses qualités d'esprit. Aussi tenait-il la foule, quoique libre, bien en main, et au lieu d'être dirigé par elle, il la dirigeait. (...) Sous le nom de démocratie, c'était en fait le premier citoyen qui gouvernait *.

[42] Selon Thomas Hobbes, Léviathan, op. cit., chapitre XVIII, p. 184, le souverain se doit "d'être juge à la fois des moyens nécessaires à la paix et à la défense, et aussi de ce qui les gène et les trouble, et de faire tout ce qu'il juge nécessaire de faire, soit par avance, pour préserver la paix et la sécurité en prévenant (...) l'bostilité à l'extérieur, soit, quand la paix et la sécurité sont perdues, pour les retrouver .

[43] Raymond Aron, Penser la guerre. Clausewitz, Gallimard, Paris, 1976, tome 2, p. 253.

[44] Citation du diplomate américain et théoricien réaliste George Kennan.

[45] Voir à ce propos R. Rosecrance, The Rise of the Trading State. Commerce and Conquest in the Modern World, Basic Pooks, New York, 1986.

[46] Aux Etats-Unis, un sondage CBS/New York Times, effectué du 5 au 7 janvier 1991, soir 10 jours avant l'ultimatum du 15 janvier, montrait ainsi que, si $48 \%$ des Américains interrogés étaient favorables à un passage à l'action des marines, $47 \%$ préféraient une attitude de "wait and see" En France, un sondage CSA/France Inter/Le Parisien datant des 7-8 janvier 1991 indique, quant à lui, que $57 \%$ des personnes interrogées étaient favorables à ce que tout devait être fait "pour négocier une solution de compromis avec le président irakien", contre $35 \%$ seulement pour qui on "ne peut accepter aucun compromis tant que les Irakiens n'auront évacué le Koweit".

[47] Voir à ce sujet les réflexions de J. Mueller, Retreat From Doomsday. The Obsolescence of Major War, Basic Books, New York, 1989.

[48] Voir, dans le même sens, E.O. Czempiel, "Governance and Democratization", dans 
J.N. Rosenau et E.O. Czempiel (sous la direction de), Governance Without Government. Order and Change in World Politics, Cambridge University Press, Cambridge (Mass.), 1992, p. 250-271.

[49] Voir à ce sujet les réflexions de A. Wendt, "The Agent-Structure Problem in International Relations Theory", International Organization, vol. 41, $\mathrm{n}^{\circ} 3$, automne 1987, p. 335-370; ainsi que "Anarchy Is What States Make of It. The Social Construction of Power Politics", International Organization, vol. 46, $\mathrm{n}^{\circ} 2$, printemps 1992, p. 391-425.

[50] Raymond Aron, Paix et guerre entre les nations, op. cit., p. 108.

[51] Francis Fukuyama, La fin de l'bistoire et le dernier homme, Flammarion, Paris, 1992, considère la démocratie représentative comme constituant le "point final de l'évolution idéologique de l'bumanité ", la "forme finale de tout gouvernement humain".

[52] Zaki Laïdi, L'ordre mondial relâcbé, op. cit., p. 38.

[53] Ibid., p. 24.

[54] C'est Henry Kissinger, Le chemin de la paix, Denoël, Paris, 1972, qui fait cette distinction entre "systèmes révolutionnaires" et " conservateurs".

[55] Cette définition de l'Etat comme "war making machine " est due au théoricien réaliste R. Gilpin, War and Change in World Politics, Cambridge University Press, Cambridge (Mass.), 1981 , p. 131.

[56] Sur le couple ami-ennemi comme catégorie fondamentale de la politique internationale, voir C. Schmitt, La notion de politique, Flammarion, Paris, 1992.

[57] Thomas Hobbes, Léviathan, op. cit., chapitre XIII, p. 126.

[58] H. Bull, The Anarchical Society. A Study of Order in World Politics, Columbia University Press, New York, 1977, p. 13

[59] Selon H. Grotius, Le droit de la guerre et de la paix, Guillaumin, Paris, 1867, Prolégomènes, paragraphe 25, "la guerre ne doit être entreprise qu'en vue d'obtenir justice et, lorsqu'elle est engagée, elle ne doit être conduite que dans les termes du droit et de la bonne foi".

[60] Selon E. de Vattel, Le droit des gens, Carnegie Institution, Washington, 1916, Livre 2, chapitre V, l'état de détente est l'exact opposé du "vaste brigandage " qui caractériserait les relations entre nations dans le cas où ne règnerait aucune justice entre elles.

[61] Voir D. Senghaas, "Weltinnenpolitik. Ansätze für ein Konzept ", Europa-Archiv, vol. 47, n' 22, 25 novembre 1992, p. 643-652.

[62] C'est la distinction proposée par K. Waltz, Theory of International Politics, op cit.

[63] C'est la distinction proposée par J. Rosenau, Turbulence in World Politics, op. cit. 